\title{
Pair Scattering of Electrons in Edge Channels of Opposite Chiralities in the Presence of a Disorder Potential
}

\author{
M. G. Prokudina, prok@issp.ac.ru, V. S. Khrapai, dick@issp.ac.ru \\ Institute of Solid State Physics, Russian Academy of Sciences, Chernogolovka, Moscow region, 142432 Russia
}

\begin{abstract}
The nonequilibrium transfer of the energy between electrons of counter-propagating quasi-one-dimensional systems has been perturbatively calculated for edge channels in a two-dimensional system in the integer quantum Hall effect. The processes involving two electrons that are allowed only in the system with disorder have been taken into account. Expressions for the cases of Coulomb scattering and transfer of nonequilibrium phonons have been obtained. The energy transferred per unit time has a quasi-threshold dependence on the degree of nonequilibrium of the hot channel. According to numerical estimates for electrons in GaAs, Coulomb scattering processes dominate in the energy transfer and the expected effect can be experimentally observed.
\end{abstract}

The momentum and energy conservation laws in the one-dimensional case impose significant constraints on electron-electron scattering. For example, the Coulomb drag effect between single-channel quantum wires is 'resonant and maximal when the interacting systems are identical [1]. In the regime of the integer quantum Hall effect, where the diagonal conductivity in the bulk of the two-dimensional system is exponentially small, chiral 'quasi-one-dimensional systems in which backscattering is suppressed (edge channels) can exist [2]. In the edge channel, all electrons have the same direction 'of the momentum (chirality sign) and drag current without transfer of particles from one channel to another cannot be detected at least for a linear dispersion law 3. Nevertheless, there is an experimental method for measuring the energy transferred in the inelastic 'scattering of electrons in different channels [4, 5].

The pair scattering of electrons in co-propagating 'edge channels is forbidden by the momentum conservation law except for the cases where the 'dispersion laws are the same or where disorder providing uncertainty in the momentum exists in a system [6]. Energy transfer between two electrons in the counter-propagating edge channels in a pure system is strictly forbidden. This concerns both the Coulomb scattering and transfer of a nonequilibrium phonon when one electron absorbs a phonon emitted by another electron. In this case, three-particle processes that are allowed in the presence of the nonlinear dispersion law should dominate [7, 8]. However, the pair interaction between counter-propagating edge channels can exist in a non-ideal system where the role of disorder is significant.

In this work, we study energy transfer between counter-propagating edge channels one of which has a nonequilibrium distribution and the second channel is initially in equilibrium in the presence of smooth disorder using the idea proposed in [6]. We consider both the direct unscreened Coulomb interaction and the interaction through the transfer of nonequilibrium acoustic phonons. Expressions for the scattering matrix elements are obtained in perturbation theory. Numerical estimates are given for edge channels in a two-dimensional system based on GaAs.

We consider two edge channels with length $L$ 9] with the linear dispersion law $E(k)=\hbar v_{F} k\left(v_{F}\right.$ is the drift velocity at the edge) at distance $d$ from each other. Channels are separated by a thin barrier impenetrable for electrons and have opposite chiralities. We assume that the integer quantum Hall effect for simplicity with one filled Landau level $(\nu=1)$ is implemented in the bulk of the two-dimensional system. The energy gap between Landau levels is the largest scale in the problem. Moreover, we neglect the modulation of the density of states at the edge and the corresponding edge features [10]. This implies that the linear dispersion law is valid for all energies at which the distribution function noticeably differs from the equilibrium distribution function.

As is known, an isolated edge channel in the integer Quantum Hall effect is described in a Fermi liquid model [11]. A system of two interacting counterpropagating edge channels represents a realization of the Luttinger liquid (LL) and is generally solved by use of a bosonization technique. Energy relaxation in the $\mathrm{LL}$ is related to a finite life-time of the bosons and is absent in a clean system [12. Nevertheless, a finite size of the interaction region gives rise to boson scattering (and energy relaxation) at the boundaries of the LL [12]. We neglect this effect compared to a disorder scattering inside the LL, which is justified given a smallness of the interaction parameter, not too weak disorder and/or not 


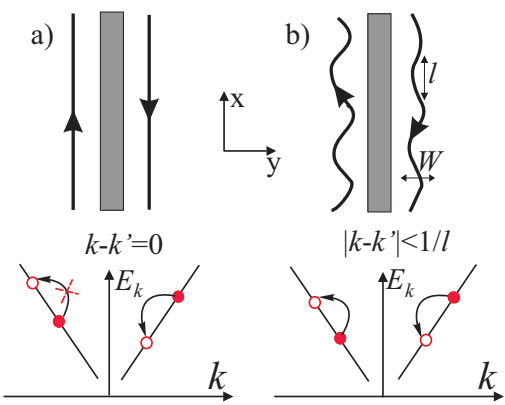

Fig. 1 Schematics of the counter-propagating edge channels separated by an impenetrable barrier and the electron spectrum for (a) an ideal system (the momentum conservation law is satisfied and pair scattering is forbidden) and (b) a system with disorder (the momentum conservation law is violated and pair scattering is allowed).

too small length of the interaction region. Below, the size of the interaction region is assumed to be small compared to the energy relaxation length (boson meanfree path) and a pertubative solution is suggested.

The momentum and energy conservation laws forbid pair Coulomb scattering in a pure system (see Fig. 1a). In the presence of smooth disorder, the equipotential surface of the edge channel is curved. As a result, uncertainty appears in the momentum and the inelastic scattering of electrons in neighboring channels becomes possible. In our model, the deviation of the edge channel from a straight line is specified by a random value $\delta d(x)$ with the correlation function $\overline{\delta d(x) \delta d\left(x^{\prime}\right)}=w^{2} \exp \left(-\frac{\left(x-x^{\prime}\right)^{2}}{2 l^{2}}\right)$, where $\quad l$ is the correlation length of smooth disorder (larger than the magnetic length) and $w$ is the characteristic deviation (see Fig. 1b). The chosen Gaussian form of the correlation function is not as important as the presence of a large enough correlation length $l$. In such a system, the wave-function of quasi-one-dimensional electrons will depend on both coordinates $x$ and $y$. Since characteristic variations of the momentum are much smaller than the inverse magnetic length $(e B /(\hbar c))^{1 / 2}$, this dependence can be written in the simplified form:

$$
\psi_{k}\left(x_{j}, y_{j}\right)=\frac{e^{-i k x_{j}}}{\sqrt{L}} f\left(y_{j}-y_{0}^{j}\left(x_{j}\right)\right)
$$

Here, subscript $j=1,2$ specifies the channel, $f$ is a narrow function normalized as $\int_{-\infty}^{+\infty} f^{2} d y_{j}=1$, and $y_{0}^{j}\left(x_{j}\right)= \pm d / 2+\delta d_{j}\left(x_{j}\right)$, where $\delta d_{j}\left(x_{j}\right)$ is the random deviation from the average value $\overline{y_{0}^{j}}= \pm d / 2$ (the upper and lower signs correspond to the first and second channels, respectively) and $\mathrm{d}$ is the average distance between the channels. Owing to this choice of the wavefunction, the matrix element of pair scattering for the unscreened Coulomb and electron-phonon interactions can be represented in an explicit form.

We denote the initial and final states of two scattered electrons as $|i\rangle=\left|k, k^{\prime \prime}\right\rangle$ and $|f\rangle=\left|k^{\prime}, k^{\prime \prime \prime}\right\rangle$, respectively. For the Coulomb interaction in a medium with the dielectric constant $\chi$, the matrix element in the first order of perturbation theory is obtained in the form:

$V_{i f}=\int \psi_{k^{\prime}}^{*}\left(r_{1}\right) \psi_{k^{\prime \prime \prime}}^{*}\left(r_{2}\right) \frac{e^{2}}{\chi\left|r_{1}-r_{2}\right|} \psi_{k}\left(r_{1}\right) \psi_{k^{\prime \prime}}\left(r_{2}\right) d r_{1} d r_{2}$

where $r_{j}$ is the coordinates of the electron in the $j$ th channel, $j=1,2$. After the substitution of the wave-functions taking into account the choice of $f(y)$, the integrand will depend only on $x_{1}$ and $x_{2}$, because $\left(y_{1}-y_{2}\right)^{2}=\left(d+\delta d_{1}\left(x_{1}\right)+\delta d_{2}\left(x_{2}\right)\right)^{2} \approx d^{2}+$ $2 d\left(\delta d_{1}+\delta d_{2}\right)$. We change the coordinates $x_{1}$ and $x_{2}$ to the relative coordinate $r_{x}$ and the coordinate of center of mass $R_{x}$. Owing to the energy conservation law, integration with respect to the relative coordinate $r_{x}$ occurs at zero momentum. We are interested in scattering with nonzero energy transfer $\varepsilon \neq 0$, where the total momentum is not conserved and the momentum transfer is $\Delta k=k+k^{\prime \prime}-k^{\prime}-k^{\prime \prime \prime}=2 \varepsilon /\left(\hbar v_{F}\right)$. The matrix element has the form:

$$
V_{i f}=\frac{e^{2} d}{\chi L^{2}} \int \frac{\delta d_{1}+\delta d_{2}}{\left(r_{x}^{2}+d^{2}\right)^{3 / 2}} e^{i \Delta k R_{x}} d R_{x} d r_{x} .
$$

where $\delta d_{j}$ depend on the both variables $R_{x}$ and $r_{x}$. It is easy to obtain an expression for the averaged square of the absolute value of the matrix element. Taking into account that $\overline{\delta d_{i}(x) \delta d_{j}\left(x^{\prime}\right)}=w^{2} \exp \left(-\frac{\left(x-x^{\prime}\right)^{2}}{2 l^{2}}\right)$ for $i=$ $j$ and 0 for $i \neq j$ because the random variables $\delta d_{1}$ and $\delta d_{2}$ are not correlated, we obtain:

$$
\overline{\left|V_{i f}\right|^{2}}=\frac{8 e^{4} w^{2} l \sqrt{2 \pi}}{\chi^{2} d^{2} L^{3}} e^{-\frac{1}{2} \varepsilon^{2} / \varepsilon_{0}^{2}}\left[\frac{|\varepsilon|}{\varepsilon_{d}} K_{1}\left(\frac{|\varepsilon|}{\varepsilon_{d}}\right)\right]^{2},
$$

Here, $K_{1}$ is the modified Bessel function of the second kind, $\varepsilon_{0}=\hbar v_{F} / 2 l$, and $\varepsilon_{d}=\hbar v_{F} / 2 d$. At $|\varepsilon| \ll \varepsilon_{d}$, the expression in square brackets is close to 1 and the asymptotic expression $\approx \sqrt{\pi|\varepsilon| / 2 \varepsilon_{d}} e^{-|\varepsilon| / \varepsilon_{d}}$ can be used for $|\varepsilon|>\varepsilon_{d}$.

When thermodynamic equilibrium in one channel is violated, energy can be transferred to a neighboring channel and the channel can be heated. Let $E, E^{\prime}$ and $E^{\prime \prime}, E^{\prime \prime \prime}$ be the initial (final) energy of the electrons in the first (nonequilibrium) and second channels, 
respectively. The energy loss of the first electron is $\varepsilon=E-E^{\prime}$ (it can formally be negative, corresponding to an increase in the energy of the first electron). We denote the distribution function in the channels at the corresponding energies as $f(k) \equiv f$ and $f\left(k^{\prime}\right) \equiv f^{\prime}$. The probability of interaction per unit time is specified by Fermi's golden rule $W_{i \rightarrow f}=\frac{2 \pi}{\hbar} \overline{\left.V_{i f}\right|^{2}} \delta\left(\varepsilon+E^{\prime \prime}-E^{\prime \prime \prime}\right)$, where $\delta$ is the Dirac delta function. In this case, the power of the energy transfer through pair scattering is:

$$
\begin{gathered}
P=\frac{2 \pi}{\hbar} \frac{L^{4}}{(2 \pi)^{4}} \iiint \int \varepsilon \overline{\left|V_{i f}\right|^{2}} f\left(1-f^{\prime}\right) \times \\
\times f^{\prime \prime}\left(1-f^{\prime \prime \prime}\right) \delta\left(\varepsilon+E^{\prime \prime}-E^{\prime \prime \prime}\right) d k d k^{\prime} d k^{\prime \prime} d k^{\prime \prime \prime}
\end{gathered}
$$

Taking into account the linear dispersion law, we transform this integration to integration with respect to the energies of scattered electrons. In addition, we denote $E^{\prime \prime \prime}-E^{\prime \prime}=\varepsilon^{\prime}$; correspondingly, $\delta\left(\varepsilon+E^{\prime \prime}-\right.$ $\left.E^{\prime \prime \prime}\right)=\delta\left(\varepsilon-\varepsilon^{\prime}\right)$. Finally, to simplify the expressions, we introduce form factors $F_{j}(\varepsilon)=\int f_{j}(E+\varepsilon)\left(1-f_{j}(E)\right) d E$ for the emission $(\varepsilon>0)$ and absorption $(\varepsilon<0)$ of the energy, where $f_{j}$ is the distribution function of electrons in the $j$ th channel. After the integration of the $\delta$-function, we obtain the final expression:

$$
\begin{aligned}
P & =\frac{2 \pi}{\hbar} \frac{L^{4}}{(2 \pi)^{4}}\left(\hbar v_{F}\right)^{-4} \times \\
& \times \int_{-\infty}^{+\infty} \varepsilon F_{1}(\varepsilon) F_{2}(-\varepsilon) \overline{\left|V_{i f}\right|^{2}} d \varepsilon .
\end{aligned}
$$

We now consider pair scattering via exchange by nonequilibrium acoustic phonons. In this case, the scattering probability amplitude should be sought in the second order of perturbation theory in the electron-phonon interaction:

$$
V_{i f}=\sum_{q_{x}, q_{y}, q_{z}} \frac{\left\langle f\left|V_{e-p h}\right| I\right\rangle\left\langle I\left|V_{e-p h}\right| i\right\rangle}{E_{i}-E_{I}+i 0},
$$

where the intermediate state $|I\rangle=\left|k^{\prime}, k^{\prime \prime}, \mathbf{q}\right\rangle$ corresponds to the relaxation of the first electron with the emission of a real or virtual phonon and summation is performed over the three-dimensional momenta of phonons and their polarizations. We assume that the phonon system is closely connected to a lowtemperature thermostat so that the steady-state phonon occupation numbers at energies of interest are much smaller than 1. Bearing in mind a GaAs crystal, we take into account the electron-phonon interaction through piezoelectric coupling, which dominates in the longwavelength limit. In the case of the emission of a phonon by an electron in the first channel and its absorption in the second channel, we obtain:

$$
\begin{array}{r}
\left\langle f\left|V_{e-p h}\right| I\right\rangle\left\langle I\left|V_{e-p h}\right| i\right\rangle=\frac{\alpha \Omega}{L^{2} V q} e^{i q_{y} d} \times \\
\times \int_{0}^{L} e^{i \Delta k_{1} x} e^{i q_{y} \delta d_{1}(x)} d x \int_{0}^{L} e^{i \Delta k_{2} x} e^{-i q_{y} \delta d_{2}(x)} d x
\end{array}
$$

where $\Omega \equiv \hbar e^{2} e_{14}^{2} /\left(2 \chi^{2} \rho s\right) ; \rho$ and $V$ are the density and volume of the crystal, respectively; and $e_{14}$ is the piezoelectric coefficient in a crystal with a zinc blende structure [13. The speed of sound $s$ and coefficient $\alpha$ depend on the direction of the momentum of a phonon and its polarization [14. Below, estimates will be given for edge channels along the [110] easy cleavage direction in a two-dimensional gas in GaAs. In this case, the main contribution comes from transverse acoustic phonons with $\alpha=0.5$ and $s=3 \times 10^{5} \mathrm{~cm} / \mathrm{s}$. The nonconserved longitudinal momentum at emission $(j=1)$ and absorption $(j=2)$ of a phonon is conveniently represented in the form $\Delta k_{j}=\varepsilon / \hbar v_{F}+(-1)^{j} q_{x}$, where the energy conservation law is taken into account. In our model, the effective width of the edge channel is $w$; consequently, the $y$ component of the phonon momentum is bounded as $\left|q_{y}\right| \lesssim 1 / w$. For this reason, the approximate expression $e^{-(-1)^{j} i q_{y} \delta d_{j}(x)} \approx 1-$ $(-1)^{j} i q_{y} \delta d_{j}(x), j=1,2$ can be used in Eq. (4). Then, Eq.(3) for the matrix element has the form:

$$
\begin{array}{r}
V_{i f}=\frac{\alpha \Omega}{8 \pi^{3} L^{2}} \int \frac{e^{i q_{y} d} d q_{x} d q_{y} d q_{z}}{q\left(\varepsilon-\varepsilon_{q}+i 0\right)}\left[q_{y}^{2} I_{1} I_{2}+\right. \\
\left.+i 2 \pi q_{y} \delta\left(q_{x}+\varepsilon / \hbar v_{F}\right) I_{1}-i 2 \pi q_{y} \delta\left(q_{x}-\varepsilon / \hbar v_{F}\right) I_{2}\right],
\end{array}
$$

where $\delta\left(q_{x} \pm \varepsilon / \hbar v_{F}\right)$ is the delta function, $\varepsilon_{q}=\hbar s q$ is the energy of the phonon, and $I_{j}=\int e^{i \Delta k_{j} x} \delta d_{j}(x) d x$. The last two terms in square brackets correspond to processes with single violation of the momentum conservation law at the emission or absorption of a phonon. The first term concerns processes where the momentum is not conserved at both the emission and absorption of a phonon. The integrals $\operatorname{Int}_{n}(\varepsilon)=$ $\int\left(q_{y}\right)^{n} e^{i q_{y} d} d q_{y} d q_{z} / q /\left(\varepsilon-\varepsilon_{q}+i 0\right)$ for $n=1,2$ should be calculated in Eq. (5). It is convenient to change the variables $d q_{y} d q_{z}=q_{\perp} d q_{\perp} d \varphi$, where $q_{\perp}=\sqrt{q_{y}^{2}+q_{z}^{2}}$ is the phonon momentum component perpendicular to the $\mathrm{x}$ axis and $\varphi$ is the angle between $\mathbf{q}_{\perp}$ and the $\mathrm{z}$ axis. Then, at $s \ll v_{F}, q_{\perp} \approx q$ because acoustic phonons under these conditions are emitted primarily along the normal to the edge channel. Changing $q \rightarrow \varepsilon_{q} /(\hbar s)$, we finally obtain:

$$
\operatorname{Int}_{n}(\varepsilon) \approx(\hbar s)^{-n-1} \int \sin ^{n} \varphi d \varphi \int \frac{\varepsilon_{q}^{n} e^{i \varepsilon_{q} \tau \sin \varphi} d \varepsilon_{q}}{\varepsilon-\varepsilon_{q}+i 0}
$$


where $\tau=d / s$ is the characteristic time of flight of a phonon between the channels. In Eq. (6), the integral with respect to the energy of the phonon over the range $0<\varepsilon_{q}<\hbar s(w|\sin \varphi|)^{-1}$ is taken first and the integral with respect to $\varphi$ is then calculated. First, we present an answer in the limit $\varepsilon \gg \hbar / \tau$; for the characteristic values $\varepsilon \sim \varepsilon_{0}$, this limit is equivalent to a long distance between the edge channels $d \gg$ $l s / v_{F}$. As will be shown below, the last condition is certainly satisfied when the electron.phonon mechanism can compete with the Coulomb mechanism. In this limit, the main contribution to the integral comes from phonons in a narrow energy band $\left|\varepsilon_{q}-\varepsilon\right| \lesssim \hbar / \tau$. As a result,

$$
\left.\operatorname{Int}_{n}(\varepsilon) \approx \frac{2 \pi^{2} \varepsilon^{n}}{(i \hbar s)^{n+1}} \frac{d^{n}}{d a^{n}}\left(J_{0}(a)+i H_{0}(a)\right)\right|_{a=\varepsilon \tau / \hbar}
$$

where $J_{0}$ and $H_{0}$ are the Bessel function of the first kind and the Struve function of the zeroth order, respectively, and the derivative of the quantity in square brackets is taken at the indicated argument. In the limit $a \rightarrow \infty$, the asymptotic expression $J_{0}(a)+i H_{0}(a) \approx$ $\sqrt{2 / \pi a} e^{i(a-\pi / 4)}$ can be used.

In the opposite limit of small distances, $\varepsilon \ll \hbar / \tau$, all phonons contribute to the integral. As a result, the approximate answer has the form

$$
\begin{aligned}
& \operatorname{Int}_{1} \approx \frac{2 i \pi(\cos (d / w)-1)}{\hbar s d} \\
& \operatorname{Int}_{2} \approx \frac{-2 \pi \sin (d / w)}{\hbar s w d}
\end{aligned}
$$

It only remains to calculate the integral with respect to $q_{x}$. For two last terms in Eq. (5), the integral is easily calculated owing to the $\delta$-function. However, for the first term, the square of the absolute value of the matrix element should first be taken and the resulting double integral with respect to $q_{x}$ and $q_{x}^{\prime}$ should then be calculated. Finally, averaging over the random distributions $\delta d_{j}$ should be performed, similar to the case of Coulomb scattering. As a result,

$$
\begin{gathered}
\overline{\left|V_{i f}\right|^{2}}=V_{1}+V_{2}, \text { where } \\
V_{1}=2^{7 / 2}\left(\frac{\alpha \Omega}{8 \pi^{3} L^{2}}\right)^{2}\left|\operatorname{Int}_{1}\right|^{2} \pi^{5 / 2} w^{2} L l e^{-\varepsilon^{2} / 2 \varepsilon_{0}^{2}} \\
V_{2}=4\left(\frac{\alpha \Omega}{8 \pi^{3} L^{2}}\right)^{2}\left|\operatorname{Int}_{2}\right|^{2} \pi^{5 / 2} w^{4} L l e^{-\varepsilon^{2} / 4 \varepsilon_{0}^{2}}
\end{gathered}
$$

Here, subscripts 1 and 2 correspond to the single and double violations of the momentum conservation law, respectively. It is noteworthy that Eqs. (8) are also valid for the transfer of a phonon from the second channel to the first one $(\varepsilon<0)$ if change $\varepsilon \rightarrow|\varepsilon|$ is made in Eq. (7). The final expression for the power transferred between the edge channels through the electron-phonon interaction can be easily obtained from Eq. (2).

It is interesting to compare the efficiencies of two interaction mechanisms. At low energies $|\varepsilon|<\varepsilon_{d}, \varepsilon_{0}$ and/or small distances between the channels, $d \lesssim l$, the matrix element for exchange by phonons is negligible compared to the Coulomb matrix element. However, the phonon contribution becomes noticeable at $d>>$ $l$ and relatively high energies $|\varepsilon|>\varepsilon_{d}$. Indeed, the energy cutoff in Coulomb matrix element (1) occurs at $|\varepsilon| \sim \varepsilon_{d} \ll \varepsilon_{0}$, i.e., much earlier than in the electron-phonon matrix element given by Eq. (8) . As a result, in the case of the strongly nonequilibrium first channel and the weakly nonequilibrium second channel (see below), the power transferred through Coulomb scattering decreases rapidly with the distance between the channels, $P_{C} \propto 1 / d^{5}$. At the same time, the asymptotic behavior of the phonon contribution is $P_{p h} \propto 1 / d$. The ratio of the powers is given by the expression

$$
\frac{P_{p h}}{P_{C}}=\frac{e_{14}^{4} v_{F}}{6 \pi^{2} \rho^{2} s^{5}}\left(1+8 \sqrt{2} \frac{v_{F}^{2}}{s^{2}} \frac{w^{2}}{l^{2}}\right) \cdot \frac{d^{4}}{l^{4}}
$$

In a clean system, one should expect that $w / l \ll 1$ owing to the high electric field at the edge. If $w / l=0.1$ is taken for estimates, the phonon contribution becomes comparable to the Coulomb one when the distance between the channels satisfies the condition $d / l \approx 10$.

Below, we consider in more detail the case of pair Coulomb scattering in the limit of small distances between the channels, $d \ll l$, where the integrand in square brackets in (1) can be taken as 1. Solution (2) makes it possible to obtain a numerical result for the power transfer for arbitrary distribution functions of electrons in the first and second channels. In the limiting case of the strongly nonequilibrium first channel and the weakly nonequilibrium second channel, the result can be represented in an explicit form. The integral with respect to the energy in Eq. (2) has cutoff at $|\varepsilon| \sim \varepsilon_{0}$. If nonequilibrium in the first channel has a noticeably larger energy scale, the energy dependence can be neglected, i.e., $F_{1}(\varepsilon) \approx F_{1}(0)$. For example, for the quasi-equilibrium distribution with the effective temperature $F_{1}(0)=k_{B} T_{1}$ (where $k_{B}$ is the Boltzmann constant). For the two-step distribution function created by a quantum contact with the applied voltage $V$ and transmission coefficient $\operatorname{Tr}: F_{1}(0)=\operatorname{Tr}(1-\operatorname{Tr}) e|V|$. 
The condition of strong nonequilibrium corresponds to the inequality $k_{B} T_{1}, e|V| \gg \varepsilon_{0}$. At the same time, when the temperature in the second channel is low, $k_{B} T_{2} \ll \varepsilon_{0}, F_{2}(-\varepsilon) \approx \varepsilon \theta(\varepsilon)$, where $\theta$ is the Heaviside step function (i.e., energy in the second channel can only be absorbed). In this limit, solution (2) has the form

$$
P_{C}=\frac{e^{4} w^{2} L}{8 \pi^{2} \hbar^{2} v_{F} \chi^{2} d^{2} l^{2}} F_{1}(0)
$$

Therefore, in the strongly nonequilibrium case, the power transferred via pair scattering processes depends linearly on the drive voltage or on the effective temperature of the hot channel [15] (this statement is valid for both interaction mechanisms and an arbitrary distance between the channels). In the other limiting case of weak nonequilibrium in both channels $\left(k_{B} T_{2} \ll\right.$ $\left.k_{B} T_{1}, e|V| \ll \varepsilon_{0}\right) P_{C} \propto\left(T_{1}\right)^{4}, V^{4}$. Thus, the dependence of the power on the overdrive voltage or the effective temperature of the hot channel has a quasi-threshold behavior with the threshold $k_{B} T_{1}, e V \sim \varepsilon_{0}$.

Finally, we estimate the scale of the effect in a relatively clean system, assuming that the initial temperature $T_{2}^{i n}$ of electrons in the second channel before interaction is low. When the distance between the channels is $d=300 \mathrm{~nm}, v_{F}=10^{7} \mathrm{~cm} / \mathrm{s}$, disorder parameters are $l=1 \mu \mathrm{m}$ and $w=50 \mathrm{~nm}$, and nonequilibrium in the first channel is created by a quantum point contact with $\operatorname{Tr}=1 / 2$, the effective temperature in the second channel at the exit from the interaction region is [16]:

$$
T_{2}^{\text {out }}=\sqrt{\frac{12 \hbar P_{C}}{\pi k_{B}^{2}}+\left(T_{2}^{i n}\right)^{2}} .
$$

For the interaction length $L=3 \mu \mathrm{m}, \mathrm{eV}=0.5 \mathrm{meV} \gg$ $\varepsilon_{0}=0.05 \mathrm{meV}$, and $T_{2}^{\text {in }}=50 \mathrm{mK}$, we obtain $T_{2}^{\text {out }} \approx$ $130 \mathrm{mK}$. Such a temperature change can be measured in practice and was possibly observed in [17.

To summarize, pair scattering of electrons of onedimensional Fermi liquids of the opposite chiralities has been calculated. The energy is transferred between systems owing to the violation of the momentum conservation law in the presence of a smooth disorder potential. Expressions for the power transfer in the case of the Coulomb and electron-phonon scattering mechanisms have been obtained for the edge channels in a two-dimensional GaAs system. At small distances between the channels, Coulomb scattering dominates and the maximum energy quantum transferred in interaction is determined by the disorder correlation length similar to 6]. As a result, the power transfer has a quasi-threshold dependence on the degree of nonequilibrium (temperature or drive voltage) in the hot channel.

We are grateful to I.S. Burmistrov, I.V. Gornyi, D.V. Shovkun, and A.A. Shashkin for stimulating discussions and to D.G. Polyakov for valuable remarks. This work was supported by the Russian Foundation for Basic Research, the Russian Academy of Sciences, the Ministry of Education and Science of the Russian Federation, and the Council of the President of the Russian Federation for Support of Young Scientists and Leading Scientific Schools (project no. MK3102.2011.2).

Список литературы

1. P. Debray, V.N. Zverev et al, Semicond. Sci. Technol. 17, R21-R34 (2002)

2. M. Buttiker, Physical Review Letters 57, 1761 (1986)

3. M. Pustilnik, M. G. Mishchenko et al, Phys. Rev. Lett. 91, 126805 (2003)

4. H. le Sueur, C. Altimiras et al, Physical Review Letters 105, 056803 (2010)

5. C. Altimiras, H. le Sueur et al, Physical Review Letters 105, $226804(2010)$

6. A. M. Lunde, S. E. Nigg, and M. Buttiker, Phys. Rev. B.81, 041311(R)(2010)

7. T. Karzig, L. I. Glazman, and F. von Oppen, Phys. Rev. Lett. 105, 226407 (2010)

8. G. Barak, H. Steinberg et al, Nature 6, 489 (2010)

9. All formulas are also valid for infinitely long edge channels interacting in the finite segment with the length $L$.

10. D. B. Chklovskii, B. I. Shklovskii, L. I. Glazman Phys. Rev. B 46, 4026 (1992)

11. M.P.A. Fisher, L. Glazman in "Mesoscopic Electron Transport NATO ASI Series E, Kluwer Ac. Publ., Dordrecht (1997)

12. D.B. Gutman, Y. Gefen, and A.D. Mirlin, Phys. Rev. B 80, 045106 (2009); D.A. Bagrets, I.V. Gornyi, and D.G. Polyakov, Phys. Rev. B 80, 113403 (2009)

13. Yu. I. Sirotin, M.P. Shaskolskaya, "Osnovy Kristallografii", M., "Nauka", 1979

14. L. Fedichkin and A. Fedorov, Physical Review A 69, 032311 (2004)

15. A linear dependence of the power transfer for threeparticle scattering processes on the overdrive voltage is possible only at $T r \ll 1$. In other cases, the dependence will be at least quadratic.

16. C. Altimiras et al., Nature Phys. 6, 34 (2010).

17. D. Sprinzak, E. Buks, M. Heiblum, and H. Shtrikman, Phys. Rev. Lett. 84, 5820 (2000) 